\title{
EDITORIAL
}

\section{Pseudomonas aeruginosa and cyanide production}

\author{
W. Lenney and F.J. Gilchrist
}

n nfection and subsequent chronic infection of the cystic fibrosis (CF) lung with organisms such as Pseudomonas aeruginosa probably occurs at an early stage in the life of a child suffering from CF. Clinicians worldwide are eager to isolate the organism as early as possible in an attempt to treat the patient before any permanent damage occurs and hopefully to eliminate the organism completely from the respiratory tract for many years. Young children are usually unable to expectorate sputum for microbiological analysis and cough swabs are relatively insensitive. Induced sputum techniques are generally not well tolerated in children and bronchoscopic manoeuvres are very invasive, requiring a general anaesthetic. There has, therefore, been interest in discovering whether there are any surrogate markers which could be helpful as a specific indication of the presence of $P$. aeruginosa. It has been postulated that cyanide could be one such compound.

$P$. aeruginosa is one of a limited number of organisms which can synthesise cyanide [1]. Synthesis occurs by the oxidative decarboxylation of glycine by a hydrogen cyanide synthase enzyme. This process also produces four electrons and four hydrogen ions per glycine molecule. Cyanide synthesis by $P$. aeruginosa occurs under microaerobic $\left(\mathrm{O}_{2}<5 \%\right)$ conditions but is inactivated under both atmospheric oxygen and strictly anaerobic conditions [1, 2]. Cyanide production by $P$. aeruginosa is maximised at temperatures between $34^{\circ} \mathrm{C}$ and $37^{\circ} \mathrm{C}$ [3]. Studies which have investigated cyanogenesis in relation to $P$. aeruginosa using liquid or solid samples have assayed cyanide concentrations using a cyanide electrode. Studies analysing gas samples have measured the volatile compound hydrogen cyanide (HCN) using mass spectrometry techniques.

In $P$. aeruginosa cultures the cyanide level has been shown to reach 300-500 $\mu \mathrm{M}[1-3]$ and in the headspace over the cultures the HCN concentration has reached $17 \mathrm{ppm}$ [4]. Cyanide has also been identified in the sputum $[5,6]$ and HCN in the breath of CF and non-CF bronchiectasis patients infected with $P$. aeruginosa $[5,7]$. Conversely, cyanide is absent from the sputum of bronchiectasis patients without $P$. aeruginosa infection $[5,6]$ and $\mathrm{HCN}$ is very low or absent from the breath of healthy children [8]. Studies in healthy adults have shown that much of the HCN is generated in the mouth and this could have importance when measuring $\mathrm{HCN}$ in exhaled breath in relation to infection with $P$. aeruginosa [9].

Keele University and University Hospital of North Staffordshire, Stoke-on-Trent, UK.

CORRESPONDENCE: W. Lenney, Academic Dept of Child Health, University Hospital of North Staffordshire, Stoke-on-Trent, ST4 6QG, UK. E-mail: w.lenney46@hotmail.co.uk
The biological reason for the presence of cyanide in association with $P$. aeruginosa is unclear but it is likely that it provides $P$. aeruginosa with an advantage in the range of ecological niches it inhabits and may, therefore, have a role in its pathogenicity [10]. This is supported by studies showing cyanide to be the mediating factor in the paralytic killing model of Caenorhabditis elegans by P. aeruginosa [11]. Cyanide is highly toxic, rapidly diffusing through tissues to irreversibly bind to the terminal oxidases of aerobic respiratory chains inhibiting aerobic respiration. $P$. aeruginosa seems to avoid the toxic effects of cyanide by active detoxification mechanisms and synthesis of a respiratory chain at the end of which is found a terminal oxidase insensitive to cyanide [12-15].

$P$. aeruginosa is one of many organisms which employ quorum sensing, a process by which extracellular molecules are used to regulate phenotypes in response to population density. In $P$. aeruginosa, cyanide production is regulated, in part, by quorum sensing, with high population densities inducing cyanide synthesis [16-18]. The high $P$. aeruginosa population density of CF mucus maximises quorum-sensing cyanide production and together with its low $\mathrm{O}_{2}$ concentration and ideal temperature, provides the perfect environmental parameters for $P$. aeruginosa cyanogenesis. The high concentrations of cyanide identified in the sputum and breath of CF patients [5-7] may well affect lung cellular function within human lungs, contributing to the long-term damage known to result from chronic $P$. aeruginosa infection. It may also assist $P$. aeruginosa in excluding other lung pathogens leading to it being the dominant bacterium.

Although high $P$. aeruginosa population densities upregulate cyanide production by quorum sensing, a study measuring the cyanide levels in the sputum of CF and non-CF patients with bronchiectasis showed cyanide levels to be independent of the $P$. aeruginosa bacterial load [11]. This and the absence of cyanide from a small number of sputum/breath samples from $P$. aeruginosa infected patients [5-7] suggest that cyanide production is dependent on the presence of specific $P$. aeruginosa strains as well as the total $P$. aeruginosa load. This theory that cyanide production varies between $P$. aeruginosa genotypes is supported by the finding that the Liverpool epidemic $P$. aeruginosa strain overproduces certain quorum sensing regulated exoproducts such as LasA protease and elastase [19]. To our knowledge, no study has yet assessed the variation in cyanide production by different $P$. aeruginosa genotypes.

It must be considered that cyanide is found in sputum and breath of patients due to cyanogenesis from nonmicrobial sources. Human leukocytes challenged with Staphylococcus epidermidis have been reported to produce HCN in vitro [20-22] 
and it is possible that the airway inflammation caused by $P$. aeruginosa infection results in cyanide production by leukocytes. If this was correct, however, any organism in the lower respiratory tract causing widespread airway inflammation would lead to cyanide production and yet, cyanide has been shown to be absent from the sputum of $\mathrm{CF}$ and non-CF bronchiectasis patients infected with organisms other than P. aeruginosa $[5,6]$.

A recent review of bacterial cyanogenesis in the CF lung [23] concluded that "the detection of cyanide and metabolites from individuals infected with $P$. aeruginosa opens the door to improved monitoring of targeted anti-pseudomonal interventions and possibility to screening for early infection and impending exacerbations using sensitive methodologies to examine exhaled breath."

In the current issue of the European Respiratory Journal, STUTZ et al. [24] have once again shown that cyanide is increased in relation to $P$. aeruginosa infection in children with CF. However, they also found increased levels of cyanide in children apparently uninfected with the organism and their results suggest that the differences between the two groups of patients were too small to be of value in the individual patient to use cyanide as a marker for the early detection/presence of $P$. aeruginosa. The authors go on to suggest there was a correlation between the level of neutrophils in the sputum samples and the level of cyanide and postulate that cyanide in their samples may be derived from the neutrophils rather than $P$. aeruginosa alone. It is known that cyanide is very water soluble and it is possible that due to their methodology, losses occurred between collection of sputum and measurement of cyanide levels. Such losses are also very dependent on the $\mathrm{pH}$ of the specimen. An interesting discussion point is what is the best methodology for accurate and meaningful detection of cyanide/HCN. We know there are differences in results obtained looking at exhaled breath condensate rather that exhaled breath [25].

Whatever, the results are very interesting and give us further information about the intriguing role that cyanide plays in relation to $P$. aeruginosa infection. It is clear that further studies are needed using novel sensitive methodologies to progress our understanding and answer once and for all whether cyanide/HCN measurement does have a role in early identification of the presence of $P$. aeruginosa and whether it can be helpful in future targeted therapies.

\section{STATEMENT OF INTEREST}

None declared.

\section{REFERENCES}

1 Blumer C, Haas D. Mechanism, regulation, and ecological role of bacterial cyanide biosynthesis. Arch Microbiol 2000; 173: 170-177.

2 Worlitzsch D, Tarran R, Ulrich M, et al. Effects of reduced mucus oxygen concentration in airway Pseudomonas infections of cystic fibrosis patients. J Clin Invest 2002; 109: 317-325.

3 Zlosnik JEA, Williams HD. Methods for assaying cyanide in bacterial culture supernatant. Lett Appl Microbiol 2004; 38: 360-365.

4 Carroll W, Lenney W, Wang T, et al. Detection of volatile compounds emitted by Pseudomonas aeruginosa using selected ion flow tube mass spectrometry. Pediatr Pulmonol 2005; 39: 452-456.
5 Ryall B, Davies JC, Wilson R, et al. Pseudomonas aeruginosa, cyanide accumulation and lung function in $\mathrm{CF}$ and non-CF bronchiectasis patients. Eur Respir J 2008; 32: 740-747.

6 Sanderson K, Wescombe L, Kirov SM, et al. Bacterial cyanogenesis occurs in the cystic fibrosis lung. Eur Respir J 2008; 32: 329-333.

7 Enderby B, Smith D, Carroll W, et al. Hydrogen cyanide as a biomarker for Pseudomonas aeruginosa in the breath of children with cystic fibrosis. Pediatr Pulmonol 2009; 44: 142-147.

8 Enderby B, Lenney W, Brady M, et al. Concentrations of some metabolites in the breath of healthy children aged 7-18 years measured using selected ion flow tube mass spectrometry (SIFTMS). J Breath Res 2009; 3: 036001.

9 Wang T, Pysanenko A, Dryahina K, et al. Analysis of breath, exhaled via the mouth and nose, and the air in the oral cavity. J Breath Res 2008; 2: 037013.

10 Williams HD, Zlosnik JEA, Ryall B. Oxygen, cyanide and energy generation in the cystic fibrosis pathogen Pseudomonas aeruginosa. Adv Microb Physiol 2007; 52: 1-71.

11 Gallagher LA, Manoil C. Pseudomonas aeruginosa PAO1 kills Caenorhabditis elegans by cyanide poisoning. J Bacteriol 2001; 183: 6207-6214.

12 Zlosnik JEA, Tavankar GR, Bundy JG, et al. Investigation of the physiological relationship between the cyanide-insensitive oxidase and cyanide production in Pseudomonas aeruginosa. Microbiology 2006; 152: 1407-1415.

13 Cipollone R, Ascenzi P, Frangipani E, et al. Cyanide detoxification by recombinant bacterial rhodanese. Chemosphere 2006; 63: 942-949.

14 Cunningham L, Pitt M, Williams HD. The cioAB genes from Pseudomonas aeruginosa code for a novel cyanide-insensitive terminal oxidase related to the cytochrome bd quinol oxidases. Mol Microbiol 1997; 24: 579-591.

15 Cunningham L, Williams HD. Isolation and characterization of mutants defective in the cyanide-insensitive respiratory pathway of Pseudomonas aeruginosa. J Bacteriol 1995; 177: 432-438.

16 Williams $\mathrm{P}$, Winzer $\mathrm{K}$, Chan WC, et al. Look who's talking: communication and quorum sensing in the bacterial world. Philos Trans R Soc Lond B Biol Sci 2007; 362: 1119-1134.

17 Girard G, Bloemberg GV. Central role of quorum sensing in regulating the production of pathogenicity factors in Pseudomonas aeruginosa. Future Microbiol 2008; 3: 97-106.

18 Bjarnsholt T, Givskov M. The role of quorum sensing in the pathogenicity of the cunning aggressor Pseudomonas aeruginosa. Anal Bioanal Chem 2007; 387: 409-414.

19 Salunkhe P, Smart CHM, Morgan JAW, et al. A cystic fibrosis epidemic strain of Pseudomonas aeruginosa displays enhanced virulence and antimicrobial resistance. J Bacteriol 2005; 187: 4908-4920.

20 Stelmaszyńska T. Formation of $\mathrm{HCN}$ and its chlorination to $\mathrm{ClCN}$ by stimulated human neutrophils -2 . Oxidation of thiocyanate as a source of HCN. Int J Biochem 1986; 18: 1107-1114.

21 Stelmaszyńska T. Formation of HCN by human phagocytosing neutrophils - 1. Chlorination of Staphylococcus epidermidis as a source of HCN. Int J Biochem 1985; 17: 373-379.

22 Zgiczyński JM, Stelmaszyńska T. Hydrogen cyanide and cyanogen chloride formation by the myeloperoxidase- $\mathrm{H} 2 \mathrm{O} 2-\mathrm{Cl}^{-}$system. Biochim Biophys Acta 1979; 567: 309-314.

23 Anderson RD, Roddam LF, Bettiol S, et al. Biosignificance of bacterial cyanogenesis in the CF lung. J Cyst Fibros 2010; 9: 158-164.

24 Stutz MD, Gangell CL, Berry LJ, et al. Cyanide in bronchoalveolar lavage is not diagnostic for Pseudomonas aeruginosa in children with cystic fibrosis. Eur Respir J 2011; 37: 553-558.

25 Cáp P, Dryahina K, Pehal F, et al. Selected ion flow tube mass spectrometry of exhaled breath condensate headspace. Rapid Commun Mass Spectrom 2008; 22: 2844-2850. 